
\begin{tabular}{l}
\hline T94 TRANSFORMING ACUTE CARE IN COPD: FINDINGS FROM A \\
NATIONAL PROGRAMME OF SERVICE IMPROVEMENT
\end{tabular}

doi:10.1136/thoraxjnl-2011-201054c.94

C Thompson, P Duncan, C Blackaby, Z Lord, 0 Okosi, H Wall, A Porter. NHS Improvement - Lung, Leicester, UK

Introduction Respiratory disease presents a significant financial burden to the UK. $40 \%$ of the cost of managing respiratory disease is spent on hospital admissions. Admissions for respiratory disease account for $12 \%$ of all medical admissions and 94000 are for exacerbation of Chronic Obstructive Pulmonary Disease (COPD). Between 1991 and 2001 admissions for COPD rose by 50\% and data suggest the admissions trend continues to increase, representing an increasing burden to the NHS. The aims of this programme of work were to reduce admissions, readmissions at 30 days, length of stay (LOS) for exacerbation of COPD and to improve the quality of patient care and the patient's experience.

Methods Twelve project sites from primary care, secondary care and community services took part in an improvement workstream. They were supported by a national programme of information sharing and peer support. Service improvement methodology was used including a series of diagnostic tools. Secondary care data were used to demonstrate areas of duplication, bottlenecks and gaps within services. The Plan Do Study Act (PDSA) cycle was used to implement changes to services and to evaluate the impact of service redesign.

Results Data from five sites demonstrate $£ 202 \mathrm{k}$ savings/cost avoidance through reductions in LOS and hospital admissions. Several important key learning points emerged from the programme of work:

- Cohorts of frequent attenders via the Emergency Department can be identified. One site identified 34 patients who accounted for 157 admissions in a 12-month period. Coordinated case management in this cohort may be effective in reducing admissions; data are not yet available to support this.

- Early access to specialist respiratory care is effective in reducing LOS. One site demonstrated a mean reduction in LOS of 0.4 days and another site a reduction of 1.5 days by instigating early specialist review.

- Improving communication and service integration is effective in reducing admissions. One site prevented 33 admissions through closer working between GP and Hospital at Home services.

Conclusion Effective service redesign can deliver improvements in the quality of respiratory services for patients with COPD and simultaneously deliver productivity gains and cost savings.

\section{P95 COST EFFECTIVENESS OF OVERNIGHT FACE-TO-FACE NURSE LED COPD SERVICE}

doi:10.1136/thoraxjnl-2011-201054c.95

D Green, 0 Hampson, S Agarwal, S Church. St Helens and Knowsley Teaching Hospitals NHS Trust, Prescot, UK

Background Prevalence of Chronic Obstructive Pulmonary Disease (COPD) is twice the UK average with emergency admission rate $175 \%$ higher in Knowsley Primary Care Trust (PCT). A primary care nurse led COPD service was initially operational from 08:00 to 10:00, thereafter telephone advice was available overnight for management of patients with exacerbation of COPD. This was later commissioned by the PCT to provide a $24 \mathrm{~h}, 365$ days face-to-face rapid response contact assessment at home or in the Accident and Emergency department. The Nurses were contracted to assess the patients within $2 \mathrm{~h}$ of the contact. Following this, staffing levels needed to be considerably increased. This development led to an increased burden on the COPD service.

Aim To assess the cost-effectiveness of the this new service provision as contracted to direct further commissioning and planning within the restricted budget.

Methods A database of patients with confirmed diagnosis of COPD was setup. The database included 3500 patients. They were provided with contact numbers in an event of exacerbation. Activity was monitored over $24 \mathrm{~h}$ from September 2009 to July 2010 (10 month's duration). Data were analysed using Excel.

Results 399 contacts made over the study period. The majority of the first contacts (18\%) were on Mondays during the day hours. Another $17 \%$ first contacts were made over the weekends. 62 patients (15\%) made contact overnight from 23:00 to 08:00 over the 10 months duration. Only 17 home visits were required and 45 patients reviewed in $A \& E$. Of the patients reviewed in $A \& E$ only five were suitable for supported discharge.

Conclusion Overnight face-to-face COPD nurse led service was not found to be cost-effective. This provides valuable information to inform the COPD national strategy consultation in development for further services.

\section{P96 A COMPARISON BETWEEN COMMUNITY BASED MODELS OF COPD CARE}

doi:10.1136/thoraxjnl-2011-201054c.96

P T Flood-Page. Aneurin Bevan Health Board, Gwent, UK

Introduction Decentralisation of healthcare commissioning in the UK has led to the development of multiple models of communitybased care for COPD. Despite the huge costs associated with these long term condition (LTC) teams there is little published data exploring the efficacy of these models of care. Until 2009 healthcare in Gwent, South Wales was delivered by five autonomous Local Health Boards (LHB). Different models of community-based COPD care were developed in each. This gave a unique opportunity to compare the efficacy of different models of healthcare among patients with similar demographic characteristics. The three main models of care were similar in primary objective: to reduce hospital admissions. In Caerphilly LHB COPD was managed by nurse specialists. In Newport and Monmouthshire generic nurses managed COPD with other chronic conditions. LTC nurses in these LHBs worked alongside GPs without secondary care. Services were introduced to some practices but not others. In Torfaen and in Blaenau Gwent COPD was managed by nurse specialists, GPs and Consultant Chest Physicians.

Methods Hospital admission data were collected from the trust database. COPD admission data were compared for the 3 years before and after the introduction of services around 2006.

Results Gwentwide 2003-2009 there was a 5.5\% increase in COPD admissions. In Caerphilly the increase was $12.3 \%$. In Newport and Monmouthshire for those practices working with LTC there was an increase of $20.8 \%$ and $43.4 \%$ respectively. This corresponded to an increase of $5.2 \%$ and $1.0 \%$ respectively in those practices in Newport and Monmouthshire that had no contact with LTC teams and continued to manage COPD in the same way $(p<0.01$ for within LHB comparison) In Blaenau Gwent and Torfaen, however, the inclusion of secondary care was associated with a reduction in admissions of $3.8 \%$ and $5.1 \%$ respectively. ( $p<0.01$ for the comparison between pooled data for Torfaen/Blaenau Gwent and Newport/ Monmouthshire/Caerphilly).

Conclusion There is little evidence base to guide the huge sums of money invested in community care for COPD and other chronic conditions. Our data suggests that services with close communication between primary and secondary care may be more effective than those developed around primary care alone. 